ORIGINAL ARTICLE

\title{
Pain outcomes in children who received intrathecal vs intravenous opioids for pain control following major urologic surgery: a retrospective review
}

\author{
Elizabeth M. Putnam, Prabhat Koppera, Shobha Malviya \& Terri Voepel-Lewis
}

Department of Anesthesiology, University of Michigan Hospital and Health Systems, Ann Arbor, MI, USA

\section{What is already known}

- Intrathecal opioid administration has been associated with short-term postoperative benefits including reduced pain and opioid use and longer interval to morphine rescue in children.

What this study adds

- Intrathecal opioids were associated with reduced need for intravenous opioids for the first $16 \mathrm{~h}$ after urologic surgery with no discernible difference thereafter. Children who received intrathecal opioids experienced higher rates of pruritus, constipation, and hypotension than those who received intravenous opioids.

\section{Keywords}

postoperative analgesia; intrathecal opioids; urologic surgery; children

\section{Correspondence \\ Dr. Elizabeth M. Putnam, Department of Anesthesiology, University of Michigan Hospital and Health Systems, 1500 East Medical Center Drive, Ann Arbor, Ml 48109, USA \\ Email: eread@med.umich.edu}

Section Editor: Per-Arne Lonnqvist

Accepted 27 August 2015

doi:10.1111/pan.12781

\section{Summary}

Background: Intrathecal (IT) opioid administration has been associated with postoperative benefits including reduced pain and opioid use in children. However, the postoperative benefits and risks of IT opioid administration during major urologic surgery in children remain unclear.

Aim: The aim of this study was to compare postoperative pain and adverse event outcomes among children who received IT vs intravenous (IV) opioids during major urologic surgery.

Methods: We reviewed the medical records of children 3-17 years of age who underwent ureteroneocystostomy or pyeloplasty between 2006 and 2012. Electronically captured anesthetic and surgical data, postanesthesia care recovery unit (PACU) and nursing flowsheets, and daily progress notes through hospital discharge were reviewed. Analgesic techniques (i.e., IT or IV patient/nurse controlled opioids), all analgesic drugs and doses were recorded. Outcome measures included pain scores, need for rescue analgesics, opioid-related adverse events, and their treatments.

Results: Seventy-seven children received IT opioids and 51 received IV opioids. More children in the IV group required rescue analgesics and had higher pain scores at PACU discharge. Children in the IV group required rescue opioids more frequently than the IT group from 0 to $8 \mathrm{~h}$ and 8 to $16 \mathrm{~h}$ after PACU discharge, but rates were similar by 16-24 h 70\% of children in IT group transitioned directly to oral opioids. Seven IT placements were considered as failed due to early need for rescue opioids. Four $(8 \%)$ of the IV group and seven $(9 \%)$ of the IT group experienced oxygen desaturation. Two of these, both in IT group required naloxone and one was admitted to ICU for observation. The IT group experienced a higher incidence of pruritus, constipation and hypotension. 
Conclusion: We observed better postoperative pain control in children who received IT vs IV opioids for the first $16 \mathrm{~h}$ with no discernible difference thereafter. The intrathecal group experienced higher incidences of pruritus, constipation, and hypotension.

\section{Introduction}

Intrathecal (IT) morphine has been used as an analgesic adjuvant during cardiac, orthopedic, abdominal, and urological surgery in children $(1,2)$. Several randomized trials have shown that children who received IT morphine intraoperatively had reduced pain and opioid requirements in the postoperative period following hypospadias repair (3), cardiac surgery (4), and spinal fusion (5). In this latter study, children had also received intravenous (IV) opioids via the patient controlled analgesia (PCA) method. Retrospective studies, in children who underwent a variety of surgeries, similarly found those who received IT morphine in addition to general anesthesia had reduced intraoperative and postoperative opioid use compared to those who received nalbuphine PCA (5-7). Studies have also found that in children who received IT opioids the interval to IV morphine rescue was longer compared to those who received intraoperative IV opioids alone $(3,4,8)$ or IT placebo $(2,6,9)$. A retrospective review of 187 children receiving low dose IT opioids for a range of major open and laparoscopic surgical procedures found that $16 \%$ of patients did not require any postoperative opioids (7).

Although the short-term postoperative benefits of IT opioid administration during surgery have been demonstrated, little is known about related adverse events. Retrospective data vary showing that pruritus, nausea, and vomiting are the most common side effects across a range of IT opioid doses with an incidence of these effects varying from $6 \%$ (3) to $35 \%$ (7). Urinary retention rates for children who received IT opioids have also varied widely, from $3 \%$ to $59 \%(6,7,10,11)$. More serious adverse effects of IT opioids include sedation and respiratory depression $(9,12)$ and postdural puncture headache $(1,7,8)$. However, randomized controlled trials comparing IT and IV opioids have found similar and low rates $(<5 \%)$ of respiratory complications in both groups $(3,4)$.

Larger, multi-center registries of anesthesiologist selfreported pediatric regional cases have, to date, included only a small proportion of cases where IT block was used $(\leq 2 \%)$, and these have reported few major adverse events (10-12). In one of these, a single case of inadvertent intravascular injection (of unspecified intrathecal medication) was reported among 506 IT injections (10), and in another, 3 of 386 cases had 'extended spinal blocks/total spinal anesthesia' (11). From the Pediatric Regional Anesthesia Network (PRAN) database which included cases from North America, only one adverse event (i.e., hypotension in an adolescent girl) was reported in 83 IT blocks (12). Although these databases suggest a very low rate of serious complications from IT injections, they do not differentiate between IT anesthesia and IT analgesia, nor give details of the drugs used. Significant adverse events are reported but rates of common side effects are not. From these self-reported registries, use of IT opioids, bupivacaine, or combined drugs could not be discerned, and thus, there are sparse data regarding outcomes related to IT analgesia.

Despite these studies, the benefits and risks of intraoperative IT morphine as they pertain to the postoperative course following major urologic surgery in children remains unclear. We, therefore, designed this study to retrospectively review and compare pain and adverse event outcomes among children who received IT vs IV opioids for postoperative pain management following major urologic surgery.

\section{Methods}

Approval was obtained from the Institutional Review Board at the University of Michigan Medical School, and a waiver of consent was granted to collect de-identifiable patient data for this study. We used our anesthesia electronic database (Centricity; General Electric Healthcare, Waukesha, WI, USA) to identify all children 3-17 years of age who underwent ureteroneocystostomy or pyeloplasty. During 2009 and 2010, the use of regional anesthesia had expanded at our institution and, specifically, IT administration of morphine became a routine practice to manage pain for children having major urologic surgery. Prior to this time, analgesia was managed using IV delivery of opioids either by PCA pump or nurse-controlled administration (NCA). For this study, we included children who underwent surgery between 2006 and 2012, to ensure a sufficient sample who had received either IT or IV opioid delivery over a period during which the surgical practices remained largely unchanged. We excluded children who received non-IT regional analgesia (i.e., epidural or caudal blocks), and children under 3 years of age (to reduce the potential for unreliable self-reported pain scores). 
Trained research assistants carefully reviewed the medical records of all subjects and recorded the following data: patient demographics, ASA physical status, surgical procedure, anesthetic technique, and routes and amounts of all intra- and postoperative opioids and adjuvant analgesics administered. Duration of induction (i.e., time from onset to incision), surgery (i.e., incision to surgical dressing time), emergence (i.e., surgical dressing completed to extubation), and hospital stay were calculated from electronically captured data.

The following outcome data were also recorded from the postanesthesia care recovery unit (PACU) and nursing flowsheets for each 8 -h period after PACU discharge for $24 \mathrm{~h}$, and during each $24-\mathrm{h}$ period thereafter until hospital discharge: highest pain scores (i.e. 0-10 numeric pain ratings), depth of sedation (i.e. University of Michigan Sedation Scores 0-4, where $0=$ awake/ alert to 4 = unrousable), and vital signs including lowest $\mathrm{SpO}_{2}$ from continuous pulse oximetry capture (routine in our setting), lowest respiratory rate (RR), heart rate (HR), and systolic blood pressure (BP).

All analgesic-related and surgical adverse events and their treatments were identified from review of flowsheets, progress notes (surgical and acute pain team), and medicine administration record through 30 days after discharge (via clinic documentation). Adverse events and their possible treatments included: pruritus (diphenhydramine), nausea/vomiting (antiemetic), oxygen desaturation and respiratory depression (naloxone and supplemental oxygen), constipation (laxatives), hypotension (defined as a $20 \%$ decrease from baseline and/or requirement of an IV fluid bolus), infection (i.e., fever with/without treatment), and postdural puncture headache (with/without blood patch treatment). All children for whom spinal analgesia was placed or attempted were included and failed IT analgesia was defined as the need for IV opioid rescue in $<8 \mathrm{~h}$ following IT injection. The medical records of children who were identified as having a failed spinal, postdural puncture headache, escalation of care or with any other questionable data underwent a secondary and independent review by one of the anesthesiologist investigators (i.e., EMP or PK) to ensure the integrity and reliability of data.

\section{Statistical analysis}

All analyses were conducted with sPss (v.21; IBM, Armonk, NY, USA) and data are presented as $n$ $(\%)$ or mean \pm standard deviation. Comparisons for nominal data (e.g., sex) were made between groups using chi-square with Fisher's exact tests. Continuous variables were compared using unpaired $t$-tests (cor- rected as appropriate based on Levene's test for equality of variance). Opioids were converted to morphine equivalents per kilogram of the child's weight and were standardized to the time spent in the hospital, for comparisons. Pain scores were treated as continuous data, and a repeated measures analysis of variance was used to compare pain scores between groups. Statistical significance was accepted if $P$ values were $<0.05$, and Bonferroni corrections were applied for the repeated measures.

\section{Results}

One hundred and fifty-eight children underwent ureteroneocystostomy or pyeloplasty during our study period; however, 30 children were either $<3$ years of age or had received an excluded regional technique. Thus, data from 128 patients were analyzed. Seventy-seven children had received IT hydromorphone (all after 2009), and 51 (96\% from 2006 to 2008), IV opioids as their primary mode of postoperative analgesia. There were no significant differences in ASA status, gender, and age or operation type between the IT and IV groups (Table 1).

\section{Perioperative data}

Table 2 presents a description of the intraoperative care of children in the groups. There was a small but significant increase in the induction time for children in the IT group. Sixty-two $(81 \%)$ children in the IT group had co-administration of bupivacaine $0.75 \%$ (mean volume $0.04 \pm 0.015 \mathrm{ml} \cdot \mathrm{kg}^{-1}$ ) and none had bupivacaine without IT opioid. Preservative-free morphine (Duramoph) was the IT opioid used in all patients and the mean dose was $4.4 \mu \mathrm{g}$ per $\mathrm{kg}$ $( \pm 0.59 \mu \mathrm{g})$. As expected, the intraoperative analgesia management of children differed significantly between the two groups (see Table 2). Additionally, in the PACU, significantly more children in the IV group required rescue opioid (Table 2).

Table 1 Characteristics of the study groups (data presented as $n$ [\%] or mean \pm SD where applicable)

\begin{tabular}{llll}
\hline & $\begin{array}{l}\text { Intrathecal } \\
(n=77)(\%)\end{array}$ & $\begin{array}{l}\text { Intravenous } \\
(n=51)(\%)\end{array}$ & $P$ value \\
\hline Age (years) & $7.24 \pm 3.40$ & $8.0 \pm 2.97$ & 0.232 \\
Female & $57(74.0)$ & $30(59)$ & 0.071 \\
ASA 1 & $20(26.0)$ & $17(33.3)$ & 0.194 \\
2 & $56(72.7)$ & $31(60.8)$ & - \\
3 & $1(1.3)$ & $3(5.9)$ & - \\
Pyeloplasty & $18(23.4)$ & $16(31.4)$ & 0.36 \\
Ureteroneocystostomy & $59(76.6)$ & $35(68.6)$ & - \\
\hline
\end{tabular}


Table 2 Description of perioperative data (presented as $n$ [\%] or mean \pm SD)

\begin{tabular}{|c|c|c|c|}
\hline & $\begin{array}{l}\text { Intrathecal } \\
(n=77)\end{array}$ & $\begin{array}{l}\text { Intravenous } \\
(n=51)\end{array}$ & $P$ value \\
\hline $\begin{array}{l}\text { Anesthesia induction } \\
\text { ( } \mathrm{min} \text { from start to end) }\end{array}$ & $43.6 \pm 13.3$ & $37.8 \pm 16.0$ & 0.027 \\
\hline $\begin{array}{l}\text { Surgery duration } \\
\text { (min from incision to } \\
\text { dressing) }\end{array}$ & $220.5 \pm 83.9$ & $227.1 \pm 75.5$ & 0.652 \\
\hline $\begin{array}{l}\text { Anesthesia emergence } \\
\text { (min from dressing end } \\
\text { to extubation) }\end{array}$ & $11.3 \pm 11.2$ & $13.8 \pm 10.9$ & 0.209 \\
\hline $\begin{array}{l}\text { Preoperative } \\
\text { acetaminophen }\end{array}$ & $20(26 \%)$ & $25(48 \%)$ & 0.010 \\
\hline \multicolumn{4}{|l|}{ Intraoperative medications } \\
\hline IV opioid administered & $29(38 \%)$ & $51(100 \%)$ & $<0.001$ \\
\hline IT bupivacaine & 62 & NA & - \\
\hline $\begin{array}{l}\text { Oral morphine } \\
\text { equivalents } \\
\text { (mean } \pm \mathrm{sD} \mathrm{mg} \cdot \mathrm{kg}^{-1} \text { ) }\end{array}$ & $0.05 \pm 0.09$ & $0.19 \pm 0.10$ & $<0.001$ \\
\hline Ketorolac & $8(10 \%)$ & $22(42 \%)$ & $<0.001$ \\
\hline Local infiltration & $17(22 \%)$ & $11(21 \%)$ & 0.901 \\
\hline \multicolumn{4}{|c|}{ Postanesthesia care medications } \\
\hline Opioids & $14(18 \%)$ & $37(71 \%)$ & $<0.001$ \\
\hline Ketorolac & $6(8 \%)$ & $5(10 \%)$ & 0.716 \\
\hline $\begin{array}{l}\text { Oral morphine } \\
\text { equivalents } \\
\left.\text { (mean } \pm \mathrm{SD} \mathrm{mg} \cdot \mathrm{kg}^{-1}\right)\end{array}$ & $0.01 \pm 0.03$ & $0.04 \pm 0.04$ & $<0.001$ \\
\hline Acetaminophen & $7(9 \%)$ & $3(6 \%)$ & 0.489 \\
\hline $\begin{array}{l}\text { PACU length of stay } \\
\text { (min) }\end{array}$ & $97.2 \pm 41.6$ & $102.7 \pm 43.2$ & 0.474 \\
\hline
\end{tabular}

PACU, postanesthesia care unit; IT, intrathecal; IV, intravenous.

\section{Postoperative analgesia outcomes}

After PACU discharge, children in the IV group received opioids via PCA/NCA pump $(n=41[80 \%])$ or intermittently as needed $(n=10[20 \%])$. In contrast, a majority in the IT group were transitioned directly to oral opioids $(n=54[70 \%])$, while fewer received PCA/ NCA $(n=12[16 \%])$ or intermittent IV $(n=9[12 \%])$ opioids. Nearly all children in the IV group $(n=49$ [96\%]) required IV opioids within the first $8 \mathrm{~h}$ after PACU discharge, compared to only $11(14 \%)$ in the IT group $(P<0.001)$. In seven $(9 \%)$ of the IT cases, the anesthesiologist had documented difficulty with the technique and these were, thus, recognized as having failed at the time of placement. Overall, the time to need for opioid rescue in the IT group was 15.6 土 7.9 h (Median 19; range 0-33; IQR 13-21 h).

The proportion of children who received opioids was also greater in the IV group from 8 to $16 \mathrm{~h}$ postoperatively (47 [92\%] vs $25[33 \%] ; P<0.001)$ but was similar by $16-24 \mathrm{~h}(46[90 \%]$ vs $70[91 \%] ; P=0.892)$. Total opioid use over the course of the hospital stay was signifi- cantly higher for the IV group compared to the IT group $(0.01 \pm 0.005$ vs $0.005 \pm 0.004 \mathrm{mg}$ oral morphine equivalents per $\mathrm{kg} \cdot \mathrm{h}^{-1}$ of stay; $\left.P<0.001\right)$. Conversely, acetaminophen use was higher for the IT group $(1.48 \pm 1.04$ vs $0.72 \pm 0.67 \mathrm{mg} \cdot \mathrm{kg}^{-1} \cdot \mathrm{h}^{-1}$ of stay; $\left.P<0.001\right)$ and ketorolac administration was not different $(0.01 \pm 0.02$ vs $\left.0.01 \pm 0.02 \mathrm{mg} \cdot \mathrm{kg}^{-1} \cdot \mathrm{h}^{-1} ; P=0.154\right)$. As shown in the Figure, average pain scores for both groups were, overall, low and there was a large and significant effect of time from baseline (PACU), where scores went up for the IT group, but did not change for the IV group (Interaction Time*Group tested with Wilks' Lambda 2.76; $P=0.032$; partial eta squared 0.324 ). There was no main effect for group (IV/IT) on pain scores in this sample $\left(F=0.498 ; P=0.484\right.$; partial $\left.\eta^{2}=0.011\right)$.

\section{Adverse events}

The overall incidence of opioid-related adverse effects was higher for the IT group (75\% vs 67\%); however, this did not reach statistical significance in this sample $(P=0.092)$ (Table 3). The incidence of bothersome but not dangerous side effects, including nausea, vomiting, and pruritus, was high in both groups. The rates of pruritus, constipation, and hypotension were significantly higher in the IT group. The rates of respiratory compromise were similar in both groups and included: Seven cases of oxygen desaturation in the IT group (9\%)-five requiring supplemental oxygen, and four $(8 \%)$ in the IV group, all requiring oxygen. Of these, two children (both in the IT group) required naloxone administration in the PACU and one was admitted to the intensive care unit for observation. Due to the clinical presentation of these patients, an inadvertent opioid overdose was suspected and documented. There was no difference in the length of hospital stay between the two groups (IT group $2.48 \pm 1.59$ days; IV group $2.74 \pm 0.41$ days; $P$ $=0.413$ ).

During the 30-day follow-up period, two children in the IT group and four in the IV group had complained of nausea and vomiting shortly after discharge from hospital. Similar numbers in both groups reported ongoing pain: seven $(9 \%)$ in the IT group and five $(10 \%)$ in the IV group. Two children in the IT group had reported headache without intervention in the early days after discharge. The documented details of headache in these cases were inconsistent with diagnosis of postdural puncture headache.

\section{Discussion}

Similar to previous studies in children, our study shows a reduction in intra- and postoperative opioid 
Table 3 Description of Adverse events ( $n[\%])$

\begin{tabular}{lccc}
\hline & $\begin{array}{c}\text { Intrathecal } \\
(n=77)(\%)\end{array}$ & $\begin{array}{c}\text { Intravenous } \\
(n=51)(\%)\end{array}$ & Pvalue \\
\hline $\begin{array}{l}\text { Overall analgesic-related } \\
\text { adverse events }\end{array}$ & $58(75)$ & $32(67)$ & 0.294 \\
Nausea \pm vomiting & $51(66)$ & $30(59)$ & 0.395 \\
$\quad$ Antiemetic & $39(51)$ & $25(49)$ & \\
Pruritus & $31(40)$ & $7(14)$ & 0.001 \\
$\quad$ Diphenhydramine & $15(20)$ & $7(14)$ & \\
Constipation requiring & $23(30)$ & $7(13)$ & 0.035 \\
laxative & & & \\
Urinary retention & $4(5)$ & $1(2)$ & 0.647 \\
Bladder spasm & $22(29)$ & $13(26)$ & 0.702 \\
Oxygen desaturation & $7(9)$ & $4(8)$ & 0.647 \\
(SpO 2 < $\%)$ & & & \\
$\quad$ Supplemental ${ }_{2}$ & $5(7)$ & $5(10)$ & 0.432 \\
Over-sedation & $2(3)$ & $1(2)$ & 1.00 \\
Naloxone given & $2(3)$ & 0 & 0.517 \\
Hypotension requiring & $14(18)$ & $2(4)$ & 0.026 \\
fluid bolus & $1(1.3)$ & 0 & 1.00 \\
Unplanned ICU admission & $2(3)$ & & - \\
Postdural headache & & & \\
\hline
\end{tabular}

use in patients who received IT opioids compared to IV only opioids $(2-4,13,14)$. A small majority of children $(53 \%)$ who received IT opioids did not require an opioid rescue until $\geq 18 \mathrm{~h}$ after surgery, and more than two-thirds transitioned directly to oral analgesics without the need for IV opioids. Pain scores were low for children in both groups and did not significantly differ. These findings suggest that IT opioid analgesia provides benefit in terms of reduced analgesic need in the early postoperative period only; we found that $16 \mathrm{~h}$ after PACU discharge, both IT and IV opioid administration were similarly efficacious in managing postoperative pain

Our finding that IT administration of opioids reduced postoperative opioid requirements during the early postoperative period is consistent with those from other studies $(4,8)$. The length of time-to-rescue in our study is consistent with other studies, ranging from 12.3 to $22.9 \mathrm{~h}$ depending on the dose of IT morphine $(3,15)$. Such data regarding the expected time to need for analgesic rescue may facilitate effective transition to oral or IV opioid or adjuvant analgesics and thereby avoid severe unrelieved pain. As seen in Figure 1, we found a consistent albeit modest increase in pain intensity in our IT group at the time we expected the IT analgesia to become ineffective. Early supplementation with nonopioid agents during the early postoperative period in our study may have resulted in only modest increase in pain scores once IT analgesia wore off with elimination of opioid need altogether in some patients.

The relatively high incidence of bothersome but nonserious adverse effects in our sample is higher than has been previously reported. Our incidence of nausea and vomiting was roughly double that found in studies $(7,8)$ where more than 100 children were followed up for $48 \mathrm{~h}$. Lower rates of nausea and vomiting (between $6 \%$ and $8 \%$ ) may reflect very short follow-up times (3) or less major surgical procedures (2). The high incidence of pruritus in our IT group is similar to that reported in other studies (7). Lower rates of pruritus (e.g., 10\%)

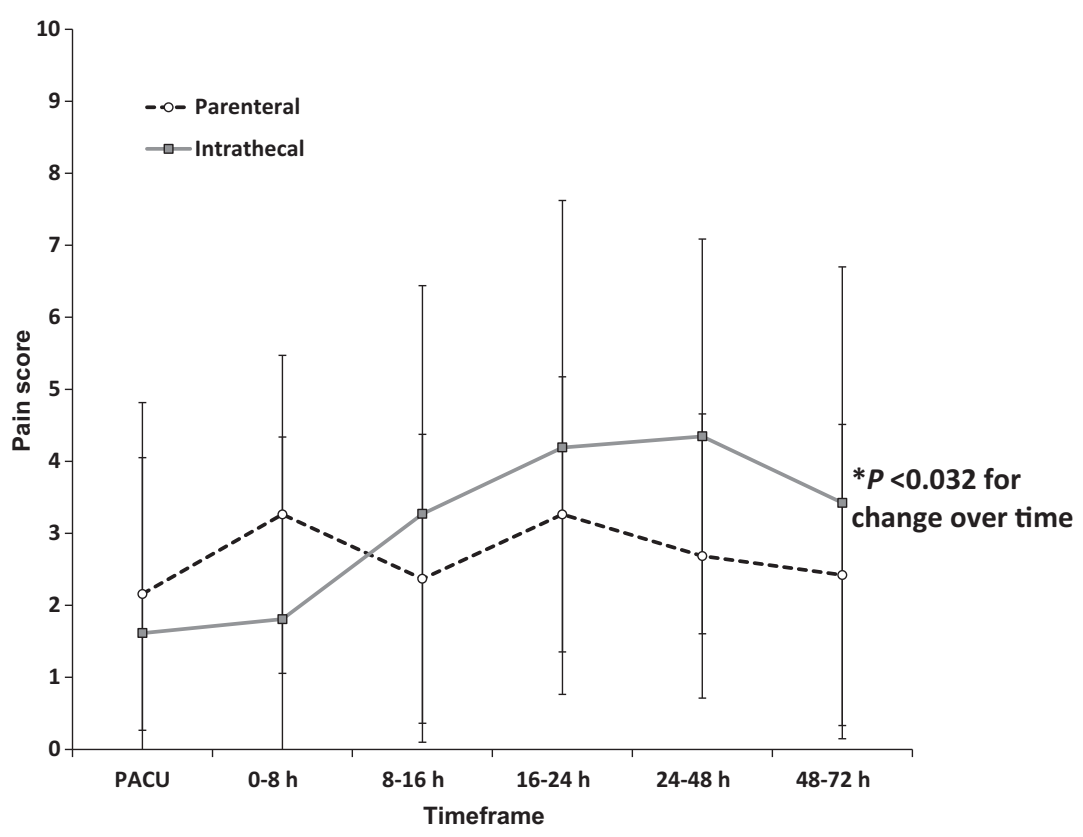

Figure 1 Reported pain scores in the groups over time. 
have been found for children treated prophylactically with naloxone infusion (6) and in those not followed beyond the early postoperative period (2). Interestingly, we found a higher rate of constipation in the IT group vs the IV group, a comparison that has not been made in previous studies. Our incidence of respiratory depression is similar to other studies with incidence varying from $1 \%$ to $10 \%$ across a range of follow-up periods. Two children in our sample required naloxone. While an overdose was suspected, these children may have been highly sensitive to opioids. This highlights the need for safety measures for children receiving opioids via any route. These should include postoperative monitoring (e.g., pulse oximetry), careful drug preparation, delivery, and 'time-outs'.

Despite somewhat equivocal findings regarding benefits and risks in our study, administering morphine intrathecally may offer some advantages over other routes of opioid administration. Firstly, the procedure itself has a clear end point (free flow of CSF) compared with other regional methods, such as epidural administration. In our study, we found a high success rate for IT analgesia (i.e., 86\%) similar to other studies (7) and the majority of failures had been identified at the time of attempted IT injection. Secondly, IT administration requires smaller opioid doses than intravenous or alternative regional routes, as it enables direct access to central and spinal cord opiate receptors (16). Overall, children in our IT group received lower doses of opioids both intraoperatively and postoperatively. In theory, lower overall opioid doses should be associated with fewer side effects; however, this was not the case. In our study and others, similar adverse effects were found when opioids are given via IT and IV routes. Thus, the opioid-sparing effect of IT opioids has, to date, not been associated with a decrease in opioid-related adverse effects and a much larger sample may be needed to demonstrate this effect. Finally, there may be a cost benefit related to the rapid transition to oral analgesia and reduced need for IV medications or PCA pump equipment during the hospital stay.

The retrospective nature of this study introduces several potential biases that may limit the interpretation of our data. Firstly, data were collected over the period where the IT technique was first introduced which may have inflated our failure rate. Additionally, our pain and adverse event outcomes were based solely on medical record documentation which introduces a significant potential of reporting bias. However, given that documentation of these outcomes is mandatory by institutional standards and captured electronically, our concern for this bias is lessened. Our study also has strengths that have not been noted previously. These include a rigorous exclusion and inclusion criteria which has not been used in previous retrospective studies. Further, we limited our sample to a large group of children operated on by the same urologic surgeons over a small time period. Generalizing our findings beyond this setting may be difficult given potential differences in surgical, anesthetic, and postoperative practices. However, similar findings in our study as in previous studies, lend external validity to our findings.

\section{Conclusions}

In summary, the intraoperative administration of IT opioids in our sample was associated with better postoperative analgesic efficacy than IV opioids alone during PACU stay and for the first $16 \mathrm{~h}$ after PACU discharge. Additionally, intraoperative administration of IT opioid was associated with a delayed need and/ or no need for intravenous opioid rescue analgesics for a majority of children. We found higher rates of the opioid-related adverse outcomes of pruritus, constipation, and hypotension in the IT group. These data can be used to inform perioperative and postoperative analgesic management for children undergoing major urologic surgery with an aim to improve safe and effective use of opioids.

\section{Ethics approval}

This work received approval from the Institutional Review Board of the University of Michigan Medical School with waiver of consent on February 12, 2012; IRB \# HUM00055997.

\section{Funding}

This research was carried out without funding.

\section{Conflict of interest}

The authors report no conflict of interest.

\section{References}

1 Jones SE, Beasley JM, Macfarlane DW et al. Intrathecal morphine for postoperative pain relief in children. Br J Anaesth 1984; 56: 137-140.
2 Apiliogullari S, Duman A, Gok F et al. Efficacy of a low-dose spinal morphine with 
bupivacaine for postoperative analgesia in children undergoing hypospadias repair. Pediatr Anesth 2009; 19: 1078-1083.

3 Suominen PK, Ragg PG, McKinley DF et al. Intrathecal morphine provides effective and safe analgesia in children after cardiac surgery. Acta Anaesthesiol Scand 2004; 48: 875-882.

4 Gall O, Aubineau JV, Berniere J et al. Analgesic effect of low-dose intrathecal morphine after spinal fusion in children. Anesthesiology 2001; 94: 447-452.

5 Tobias JD, Mateo C, Ferrer MJ et al. Reyes de Castro L. Intrathecal morphine for postoperative analgesia following repair of frontal encephaloceles in children: comparison with intermittent, on-demand dosing of nalbuphine. J Clin Anesth 1997; 9: 280-284.

6 Krechel SW, Helikson MA, Kittle D et al. Intrathecal morphine (ITM) for postoperative pain control in children: a comparison with nalbuphine patient controlled analgesia (PCA). Paediatr Anaesth 1995; 5: $177-$ 183
7 Ganesh A, Kim A, Casale P et al. Low-dose intrathecal morphine for postoperative analgesia in children. Anesth Analg 2007; 104: 271-276.

8 Tripi PA, Poe-Kochert C, Potzman J et al. Intrathecal morphine for postoperative analgesia in patients with idiopathic scoliosis undergoing posterior spinal fusion. Spine (Phila Pa 1976) 2008; 33: 2248-2251.

9 Nichols DG, Yaster M, Lynn AM et al. Disposition and respiratory effects of intrathecal morphine in children. Anesthesiology 1993; 79: 733-738; discussion 25A.

10 Giaufre E, Dalens B, Gombert A. Epidemiology and morbidity of regional anesthesia in children: a one-year prospective survey of the French-Language Society of Pediatric Anesthesiologists. Anesth Analg 1996; 83: 904-912.

11 Ecoffey C, Lacroix F, Giaufre E et al. Epidemiology and morbidity of regional anesthesia in children: a follow-up one-year prospective survey of the French-Language Society of Paediatric Anaesthesiologists (ADARPEF). Pediatr Anesth 2010; 20: 1061-1069.
12 Polaner DM, Taenzer AH, Walker BJ et al. Pediatric Regional Anesthesia Network (PRAN): a multi-institutional study of the use and incidence of complications of pediatric regional anesthesia. Anesth Analg 2012; 115: 1353-1364.

13 Hammer GB, Ramamoorthy C, Cao H et al. Postoperative analgesia after spinal blockade in infants and children undergoing cardiac surgery. Anesth Analg 2005; 100: 1283-1288, table of contents.

14 Batra YK, Lokesh VC, Panda NB et al. Dose-response study of intrathecal fentanyl added to bupivacaine in infants undergoing lower abdominal and urologic surgery. Pediatr Anesth 2008; 18: 613-619.

15 Schwartz RE, Pasquariello CA, Schlichting C. Intrathecal morphine for pediatric renal transplant recipients. Pediatr Surg Int 1994; 9: 571-573.

16 Yaksh TL, Rudy TA. Analgesia mediated by a direct spinal action of narcotics. Science 1976; 192: 1357-1358. 